Revista

\title{
Multi-Ensayos
}

Vol. 6, núm. 11

ISSN: 2412-3285

https://multiensayos.unan.edu.ni

DOI: https://doi.org/10.5377/multiensayos.v6i11.9281

\section{Evaluación en línea}

\section{Online assessment}

Marlene Rizo Rodríguez ${ }^{1}$

Recibido: 22 de octubre de 2019. Aceptado: 14 de enero de 2020

\section{RESUMEN}

La evaluación es uno de los factores más importantes en un proceso de aprendizaje, por medio de ésta se comprueban los logros alcanzados, se identifican las competencias y habilidades que han alcanzado los estudiantes en relación con los objetivos de las asignaturas. En el caso de la evaluación en línea (e-Learning) tiene cierta particularidad, dado que se hace uso de las herramientas tecnológicas. Por ello, es importante precisar las estrategias a utilizar, según la finalidad de la misma. Este ensayo, surge a partir de la experiencia como facilitadora de cursos en línea mediante la plataforma MOODLE de la asignatura Informática Básica que se imparte en todas las carreras que se ofertan en la Facultad Regional Multidisciplinaria, Estelí, UNAN-Managua. Se destacan brevemente conceptos básicos relacionados con la evaluación de aprendizajes en línea, funciones de la evaluación, la evaluación en línea, características principales, instrumentos para evaluar en línea, así como la experiencia en MOODLE.

Palabras claves: evaluación en línea; herramientas tecnológicas; MOODLE.

\section{ABSTRACT}

Evaluation is one of the essential factors in the learning process. It means that students' achievements, competencies, and skills are contrasted with the objectives of a discipline. Online evaluation (e-Learning) has some particularities since technological tools are used. It is, therefore, essential to specify the strategies to be used, depending on the academic purpose. This essay arises from experiences as a facilitator of online courses through the MOODLE platform of the general online course Basic Computing to students of the Regional Multidisciplinary Faculty, Estelí, UNANManagua. The following essay briefly highlights basic concepts related to online learning assessment, assessment functions, core features, online evaluation tools, as well as an experience using MOODLE experience.

Keywords: online evaluation; technological tools, MOODLE

1 Docente UNAN-Managua/FAREM-Estelí. Correo electrónico: mrrodriguez08@yahoo.es. (c) 2020 Revista Multi-Ensayos. 


\section{INTRODUCCIÓN}

Ante las nuevas exigencias de metodologías de la enseñanza en la Educación Superior, la Universidad Nacional Autónoma de Nicaragua, UNAN - Managua, está adaptando cursos para desarrollar algunas asignaturas en línea mediante la plataforma MOODLE, teniendo presente lo que establece el Modelo Educativo (2011) que "el proceso de enseñanza- aprendizaje se centra en el estudiante y en el desarrollo de procedimientos, habilidades, estrategias y técnicas que les permitan aprender a ser, aprender a conocer, aprender a hacer, aprender a convivir, aprender a emprender y aprender a crear" (págs. 31-32).

Partiendo de que el estudiante es el centro del proceso de enseñanza-aprendizaje, los docentes debemos preguntarnos constantemente si han aprendido, es decir, debemos estar evaluando de manera permanente el desarrollo del proceso, aunque sea un asunto difícil de obtener respuestas precisas, porque siempre nos guiamos con los resultados obtenidos de la tarea evaluadora, por tanto, sí los resultados son altos damos por hecho que son buenos, y que satisfactoriamente los estudiantes han aprendido, en tanto, si son bajos creemos que los aprendizajes de los estudiantes no son suficientes y agregamos, por qué no estudian, porque no entregan tareas, entre otros posibles argumentos. Ante estas situaciones, lo ideal es que los docentes revisemos sí las estrategias de evaluación han sido correctas y qué otro factor puede estar incidiendo para obtener esos resultados. Otro aspecto importante a destacar es que debemos estar claros que la evaluación tradicional ha sido contestar un examen, en pocas ocasiones se tenía en cuenta otra estrategia para evaluar.

En estos tiempos la tecnología ha jugado un papel importante, ya que las instituciones educativas han realizado algunos cambios en el sistema de evaluación en las clases presenciales y en línea. Ente ensayo gira alrededor de lo que aportan las tecnologías al sistema de evaluación, en este caso, realizada con la plataforma virtual MOODLE.

\section{DESARROLLO}

\section{Concepto de evaluación y evaluación en línea}

Existen diferentes conceptos sobre evaluación, en este espacio se mencionan algunos de ellos:

Díaz Barriga, (2006) la concibe como el proceso donde el alumno demuestra ciertas conductas o habilidades de acuerdo al entorno situado. Para lograr esto, indica que el docente debe emplear una gama variada de estrategias evaluativas, que le permitan obtener evidencias de las competencias adquiridas por los estudiantes.

Según Alfaro (2011), la evaluación es un proceso a través del cual se verifica el cumplimiento de los objetivos de una asignatura, módulo o curso, y determina el nivel de conocimientos, procedimientos y actitudes logrado por el estudiante. Asimismo, la función de la evaluación es retroalimentar, tomar decisiones y reforzar para motivar al estudiante a que desarrolle un conjunto de habilidades y destrezas con la finalidad que logre competencias profesionales y/o laborales. 
En tanto, Cano García, (2008) refiere que es un proceso que utiliza diversidad de instrumentos e implica a diferentes agentes, con el propósito de proporcionar información sobre la progresión en el desarrollo de la competencia y sugerir caminos de mejora.

Tal y como lo destacan los autores mencionados anteriormente, lo fundamental en un proceso de evaluación es la retroalimentación, que sirve tanto al estudiante, quien se incentiva para mejorar, y al docente que recoge la información necesaria para analizarla cuidadosamente. Además que permite que busque otras estrategias que hagan frente a las dificultades que se están presentando en el proceso de aprendizaje en el tiempo oportuno.

En relación a la evaluación en línea, se define como un proceso integral, sistemático, gradual y continuo que valora los cambios producidos en la conducta del estudiante y la eficacia de las técnicas empleadas, la capacidad científica y pedagógica de la interfaz, y todo cuanto converge en la realización del software educativo (Alfaro Saavedra, 2013).

Por tal razón, para que los docentes evalúen al inicio, durante y al final del proceso y que lo asuman con la calidad que se demanda, las instituciones educativas deben capacitar a los mismos, así como garantizar los requerimientos científicos, pedagógicos y técnicos para el buen funcionamiento de una educación en línea.

\section{Funciones de la evaluación}

En la evaluación de los aprendizajes siempre se deben considerar las funciones diagnóstica, formativa y sumativa:

En relación a la evaluación diagnóstica se realiza al inicio del curso, la que indica el nivel de conocimientos que presentan los participantes, en el caso de la modalidad virtual, permite conocer el uso de las tecnologías. Por su parte, la evaluación formativa es para documentar los avances de los estudiantes, se destacan fortalezas de los participantes en lugar de sus debilidades y se brinda retroalimentación para mejorar. En tanto, la evaluación sumativa se realiza, por lo general, al final del curso con el fin de darle una calificación al aprendizaje alcanzado.

Con respecto a esta última, considero importante señalar que, aunque muchas veces el docente la concibe como un elemento final en el proceso de aprendizaje, los estudiantes buscan cómo satisfacer contestando los puntos que contienen los exámenes, algunos el fin es aprobar, es decir, obtener la nota mínima; en cambio otros tienen la misión de valorar los conocimientos que han adquirido en el proceso de aprendizaje.

\section{La evaluación en línea}

Una gran ventaja que posee la evaluación a través de Internet, según lo indica (Cabreiros Álvarez, 2010) citando a (Fontán 2004: 6), es que "cuenta con innumerables fuentes de información para evaluar: 
resultados de pruebas objetivas, grado de cumplimiento de las actividades y tareas fijadas, actividad desarrollada en trabajos en grupo, en foros y debates, el uso y calidad de las webs que el alumno ha visitado, etc."

En el caso de la evaluación utilizando la plataforma MOODLE, desde mi experiencia como facilitadora de cursos, he tratado de calificar la participación activa de los estudiantes en las distintas actividades planteadas que van desde un foro, tareas, elaboración de wikis colaborativas, cuestionarios, hasta trabajos finales del curso. Todas estas formas de evaluar son flexibles, pero se realizan con la exigencia y calidad que se requiere, como lo estipula el Modelo Educativo de la UNAN-Managua.

\section{Características de una evaluación en línea}

Como se mencionaba anteriormente, la evaluación se ha venido transformando, no solamente se evalúa de forma tradicional, también está la evaluación en línea que tiene tres características principales. Según (Quesada Castillo, 2006) citando a (Churchill, 2004) refiere que la primera está mediada por la computadora, la segunda por la comunicación que no es en tiempo real, y tercero que cuenta con un conjunto de apoyos disponibles en línea. Es decir, depende en gran parte del uso de las tecnologías de la información y la comunicación.

\section{Instrumentos para evaluar en línea}

Existentes diferentes herramientas o instrumentos para evaluar en línea, entre ellas están:

El portafolio electrónico que se concreta cuando los estudiantes crean una estructura de carpeta y van guardando las actividades realizadas e integrando la retroalimentación que el docente les indica para mejorar, es decir, es una estrategia de evaluación flexible, asimismo, el docente puede evidenciar la gestión de la información y los conocimientos que los estudiantes han interiorizado en el proceso de aprendizaje.

Otra herramienta bastante utilizada son las rúbricas, que permiten establecer una serie de criterios que deben cumplir las actividades desarrolladas por los estudiantes.

Asimismo, existen otras formas de evaluación como son los cuestionarios, también son flexibles porque se pueden establecer varios intentos para que los estudiantes tengan la oportunidad de identificar las dificultades y corregirlas en el momento oportuno. También las mismas actividades de aprendizaje son evaluadas de manera diagnóstica y formativa. Es preciso, destacar que todos estos recursos de evaluación son confiables, la evaluación es más integral, donde los docentes evalúan el aprendizaje y no se centran en una sola calificación numérica. 


\section{Desde mi experiencia, evaluando en MOOLE}

Este proceso lo he realizado desde la asignatura Informática Básica que se contempla en el plan de estudios 2013 en las carreras que se ofertan en la UNAN-Managua, FAREM-Estelí donde laboro. El curso está diseñado para desarrollarse entre 13 a 14 semanas. Cada semana tiene diseñada tres partes principales: los recursos didácticos, las actividades de aprendizaje y el foro de consultas. Las actividades están orientadas a realizarse de manera individual y colaborativa. El proceso de evaluación es integral, se realiza en cada una de las actividades ya sea de manera diagnóstica, formativa y sumativa.

He utilizado varios de los instrumentos que proporciona la plataforma MOODLE para evaluar, ya que son fáciles de integrar, tanto para el docente como para el estudiante; además permite calificar de manera cualitativa y cuantitativa. Asimismo, puedo destacar que una de las bondades de realizar este proceso en línea, es que me ha concedido una continua y rica interacción con los estudiantes.

Otro aspecto muy importante, que me atrevo a afirmar que se tiene mayor objetividad en el proceso evaluación en línea, es la retroalimentación inmediata en cuanto a la calificación alcanzada, pues se identifican las dificultades que tienen los estudiantes, éstas se dan a conocer de inmediato y a la vez se les proporciona la forma, ideas o pistas para corregir las mismas. Con esto he logrado una relación cercana con los participantes, quienes en su mayoría están atentos para conocer sus resultados, también me ha permitido llevar un control ordenado del cumplimiento y aprendizaje que van adquiriendo los estudiantes, de igual manera ellos conocen el desempeño que han tenido en el transcurso del proceso.

\section{CONCLUSION}

La evaluación en línea es un proceso sistémico, está sustentado en el Modelo Educativo de cada institución educativa, por tanto, los facilitadores deben buscar las estrategias adecuadas así como las herramientas idóneas para alcanzar los objetivos y competencias establecidos en los programas de asignaturas.

La evaluación no es un proceso fácil, más sí es en línea, sí es importante resaltar la objetividad de los instrumentos de evaluación que se utilizan, los beneficios que se obtienen como: la mejora en la relación del facilitador con los participantes, es un proceso transparente, imparcial, se percibe el desempeño de los estudiantes de manera continua, entre otras. A la vez, que los estudiantes pueden reflexionar sobre su propio aprendizaje en cuanto a los logros traducidos en habilidades, destrezas y también de las debilidades que tienen de manera clara y precisa.

\section{REFERENCIAS}

Alfaro Saavedra, M. N. (2013). Evaluación del aprendizaje en línea. EduTiclnnova, 12-16. Recuperado el 4 de junio de 2019, de https://www.aulavirtualusmp.pe/ojs/index.php/eduticinnova/article/ view/78/0

Cabreiros Álvarez, E. (2010). La plataforma MOODLE y la evaluación de los aprendizajes. España. 
Recuperado el 3 de julio de 2019, de https://www.uoc.edu/symposia/dret_tic2011/pdf/4. cebreiros_alvarez_eduardo.pdf

Cano García, M. E. (2008). La evaluación por competencias en la educación superior. Revista de currículum y formación del profesorado, 1-16. Obtenido de https://www.ugr.es/ recfpro/ rev123COL1.pdf

Díaz Barriga, F. (2006). Enseñanza situada: vínculo entre la escuela y la vida. México: Mc Graw Hill. Quesada Castillo, R. (2006). Evaluación del aprendizaje en la educación a distancia. RED Revista de Educación a Distancia. Obtenido de https://www.um.es/ead/red/M6/quesada.pdf

UNAN, M. (2 de septiembre de 2011). Modelo Educativo, Normativa y Metodología para la Planificación Curricular. Managua, Nicaragua. Obtenido de www.unan.edu.ni 